\title{
Exploitation of the broad specificity of the membrane-bound isoenzyme of lactate dehydrogenase for direct selection of null mutants in Neisseria gonorrhoeae
}

\author{
Anne T. Hendry, ${ }^{1}$ Raj K. Bhatnagar, ${ }^{2}$ K. T. Shanmugam ${ }^{2}$ and Roy A. Jensen ${ }^{2 *}$ \\ ${ }^{1}$ Department of Laboratory Medicine, Hamilton General Hospital, 237 Barton Street East, Hamilton, Ontario, Canada \\ L8L 2X2, and Department of Pathology, McMaster University, Hamilton, Ontario, Canada L8N 3Z5 \\ ${ }^{2}$ Department of Microbiology and Cell Science, University of Florida, Gainesville, Florida 32611, USA
}

(Received 6 September 1989; accepted 21 September 1989)

\begin{abstract}
Lactic acid is readily utilized as a carbon and energy source by Neisseria gonorrhoeae. The oxidation of lactate is coupled to electron transport via a membrane-bound lactate dehydrogenase (iLDH) which is independent of pyridine nucleotide. The broad substrate specificity of iLDH endows $N$. gonorrhoeae with the novel ability to convert phenyllactate to L-phenylalanine via phenylpyruvate. $N$. gonorrhoeae ATCC 27628 typifies a class of clinical isolate whose growth is inhibited by phenylpyruvate (or L-phenylalanine). Exploiting resistance to growth inhibition by phenyllactate as a strategy of positive selection, mutant derivatives of strain ATCC 27628 lacking iLDH activity were readily obtained. These mutants are incapable of oxidizing phenyllactate, and lack the parentstrain ability to reduce $c$-type cytochromes in the presence of lactate, phenyllactate or 4-hydroxyphenyllactate. They retain, however, a cytoplasmic $\mathrm{NAD}^{+}$-linked lactate dehydrogenase (nLDH). Since the mutants retained the ability to grow on lactate as a sole source of carbon, nLDH presumably can function in an opposite-to-normal physiological direction in the absence of iLDH. This would explain the failure to isolate iLDH-deficient mutants by selection for inability to grow on lactate.
\end{abstract}

\section{Introduction}

A role for lactate dehydrogenase has been implicated in a pathogenic mechanism whereby host-derived lactate is coupled with increased gonococcal oxygen consumption at the expense of oxygen utilization by neutrophils (Britigan et al., 1988). A membrane-bound unidirectional, pyridine-nucleotide-independent lactate dehydrogenase, denoted iLDH by the nomenclature of Garvie (1980), couples lactate utilization to electron transport. An obvious approach to test the hypothesis for a role of lactate dehydrogenase in pathogenesis would be to utilize gonococcal mutants lacking iLDH activity. However, mutants unable to utilize lactate as sole source of carbon and energy have not been described. This could be explained if iLDH isoenzymes exist, e.g. an L-lactatespecific iLDH and a D-lactate-specific iLDH (which would have to be coupled with racemase activity since only L-lactate is available from host tissues). A more

\footnotetext{
Abbreviations: iLDH, membrane-bound lactate dehydrogenase independent of pyridine nucleotide; nLDH, soluble lactate dehydrogenase dependent upon pyridine nucleotide.
}

likely possibility is that the soluble pyruvate reductase, denoted nLDH in accordance with Garvie (1980), can operate in the lactate-to-pyruvate direction in the absence of iLDH.

We recently found (Bhatnagar et al., 1989a) that gonococcal iLDH possesses an unusual breadth of specificity that allows the utilization of phenyllactate and 4-hydroxyphenyllactate as alternative substrates. Thus, iLDH endows Neisseria gonorrhoeae with the unusual ability to transform phenyllactate and 4hydroxyphenyllactate to L-phenylalanine and L-tyrosine (via phenylpyruvate and 4-hydroxyphenylpyruvate), respectively.

The broad specificity of iLDH suggested to us a novel strategy for isolation of iLDH-deficient mutants, utilizing a common type of clinical isolate whose growth is inhibited in the presence of L-phenylalanine or phenylpyruvate (Hendry \& Dillon, 1984) as the starting parental strain. The biochemical and enzymological basis for this Phe $^{\mathrm{s}}$ phenotype has been reported (Bhatnagar et al., 1989b). We anticipated that mutants which are resistant to growth inhibition by phenyl- 
lactate, but which still retain the sensitivity to phenylpyruvate and L-phenylalanine manifested by the phenylalanine/phenylpyruvate/phenyllactate-sensitive parent, would be iLDH-deficient mutants. The successful implementation of this strategy is the subject of this report.

\section{Methods}

Parent strain and culture conditions. Neisseria gonorrhoeae ATCC 27628 is a clinical isolate of the type that is sensitive to growth inhibition by L-phenylalanine (Hendry \& Dillon, 1984). Cell populations for mutagenesis and routine subculturing were proliferated as confluent lawns on defined agar medium containing $0.05 \%$ fatty-acidfree bovine serum albumin (Hendry, 1983). Sterile swabs were used to spread the inoculum on plates. Newly inoculated plates were incubated in an atmosphere of $5 \%(\mathrm{v} / \mathrm{v}) \mathrm{CO}_{2}$ in air at $37^{\circ} \mathrm{C}$. Cells for extract preparation were grown in the chemically defined medium of Hendry (1983).

Mutant selection and isolation. Sterile paper discs impregnated with $10 \mu \mathrm{l}$ of a solution of ethyl methane sulphonate (EMS; Sigma) were placed in the centre of agar plates containing $0.25 \mathrm{~mm}$-phenyllactate and seeded with a confluent lawn of isolate ATCC 27628. Phenyllactate-resistant mutants were purified by three rounds of single-colony isolation on selective medium. These mutants were screened for retention of the parental sensitivity to growth inhibition by L-phenylalanine or phenylpyruvate.

Growth-rate determinations. Growth of the culture in liquid medium at $37^{\circ} \mathrm{C}$ was monitored as optical density increase by use of a KlettSummerson colorimeter (no. 66 filter). An inoculum from an exponentially growing population was used to bring $20 \mathrm{ml}$ of fresh medium at $37^{\circ} \mathrm{C}$ to a turbidity of $20-30 \mathrm{Klett}$ units. These cultures were grown in side-arm flasks at $37^{\circ} \mathrm{C}$ with gyratory shaking at 300 r.p.m. In these experiments, one Klett unit corresponds to about $10^{6}$ c.f.u. $\mathrm{ml}^{-1}$.

Extract preparation. Cells from one-litre cultures grown in defined medium (in $2 \cdot 8$-litre Fernbach flasks at $37^{\circ} \mathrm{C}$ ) were harvested by centrifugation during the exponential phase of growth. The cell pellet was washed twice with $20 \mathrm{~mm}$-potassium phosphate buffer ( $\mathrm{pH} \mathrm{7.0)}$ containing $1 \mathrm{~mm}$-dithiothreitol and resuspended in the same buffer. Cells were broken by sonication $(2 \times 30 \mathrm{~s}$ treatments at $100 \mathrm{~W}$ power output) with intermittent cooling, as needed. The resulting extract was centrifuged at $150000 \mathrm{~g}$ for $60 \mathrm{~min}$. The pellet was resuspended in the above buffer by means of a Teflon tissue homogenizer. Membrane components were solubilized at room temperature with $2 \%(\mathrm{v} / \mathrm{v})$ Triton $\mathrm{X}-100$ and $10 \mathrm{mM}-\mathrm{MgCl}_{2}$ for $20 \mathrm{~min}$. The suspension was centrifuged at $150000 \mathrm{~g}$ for $60 \mathrm{~min}$ to sediment Triton-X-100-insoluble material. The clear supernatant was used as the source of iLDH enzyme. The iLDH was assayed by a modification (Bhatnagar et al., 1989a) of the procedure of Weiner \& Heppel (1972). Protein concentration was estimated by the method of Bradford (1976).

Assay of cytochrome reduction. Cell populations in the midexponential phase of growth were harvested by centrifugation at $20000 \mathrm{~g}$ for $15 \mathrm{~min}$ at $4^{\circ} \mathrm{C}$, washed twice with $20 \mathrm{~mm}$-potassium phosphate buffer ( $\mathrm{pH} 7.0$ ) containing $1 \mathrm{~mm}$-dithiothreitol, and resuspended in the same buffer. The cells were disrupted by sonication $(3 \times 30 \mathrm{~s}$ treatments at $100 \mathrm{~W}$ power output) with intermittent cooling. The resulting suspension was centrifuged at $150000 \mathrm{~g}$ for $1 \mathrm{~h}$. The membrane-rich pellet was resuspended in a small volume of $20 \mathrm{~mm}$ potassium phosphate buffer. The total cytochrome component of the membranes was oxidized with potassium ferricyanide (approximately
$1 \mathrm{mg} \mathrm{ml}{ }^{-1}$ ), and the difference spectrum of the sample was determined using the native and ferricyanide-oxidized samples. This spectrum indicated that some of the components in the membrane vesicles were not completely oxidized during the preparation of the vesicles. In order to completely oxidize this pool, and to remove any endogenous substrates still present in the vesicles, air was passed through the vesicles at a rate of about $10 \mathrm{ml} \mathrm{min}^{-1}$, for $10 \mathrm{~min}$. These aerated samples were either oxidized by ferricyanide or reduced with sodium dithionite (about $3 \mathrm{mg} \mathrm{ml}^{-1}$ ). Both these compounds were added as solids to avoid any increase in volume and dilution. The spectral characteristics were derived immediately. When lactate, phenyllactate or hydroxyphenyllactate was used to reduce the cytochromes, these compounds were added to $1 \mathrm{ml}$ of the preparation to give a final concentration of $5 \mathrm{~mm}$. After incubation at room temperature for $5 \mathrm{~min}$ the spectra were obtained. Room-temperature difference spectroscopy and cytochrome reduction were performed using an SLM-Aminco dual-wavelength double-beam spectrophotometer.

\section{Results}

\section{Isolation of phenyllactate-resistant mutants}

Survivors of EMS mutagenesis, carried out as described in Methods with a culture of $N$. gonorrhoeae ATCC 27628, were plated out on defined medium containing a growth-inhibitory concentration of phenyllactate. Resistant colonies were readily obtained and purified by repetitive single-colony isolation. From those phenyllactate-resistant mutants which retained the parental sensitivity to growth inhibition by L-phenylalanine or phenylpyruvate, three mutants were selected for further study. Data presented in this paper for mutant ATCC 27628-1 are representative of results obtained with the other mutants.

The potency of L-phenylalanine, phenylpyruvate and phenyllactate as growth inhibitors of isolate ATCC 27628 is illustrated by disc diffusion methodology in Fig. $1(a)$. By comparison, the total resistance of mutant ATCC 27628-1 to phenyllactate is shown in Fig. $1(b)$.

Fig. 2 shows a comparison of the ability of the parent strain and its mutant derivative to grow in a defined glucose-based liquid medium in the presence of $\mathrm{L}$ phenylalanine, phenylpyruvate or phenyllactate. The single difference in the results obtained is the complete absence of effect of $100 \mu \mathrm{g}$ phenyllactate $\mathrm{ml}^{-1}$ upon the growth rate of the mutant (about 60 min doubling time), compared to the near-complete inhibition of growth seen in the parent strain ATCC 27628.

\section{Inability of mutants to utilize 4-hydroxyphenyllactate}

The growth inhibition by L-phenylalanine/phenylpyruvate/phenyllactate is due to biochemical events which cause starvation for L-tyrosine (Bhatnagar et al., $1989 b$; Hendry \& Dillon, 1984). Thus, L-tyrosine or its immediate precursor, 4-hydroxyphenylpyruvate, will 

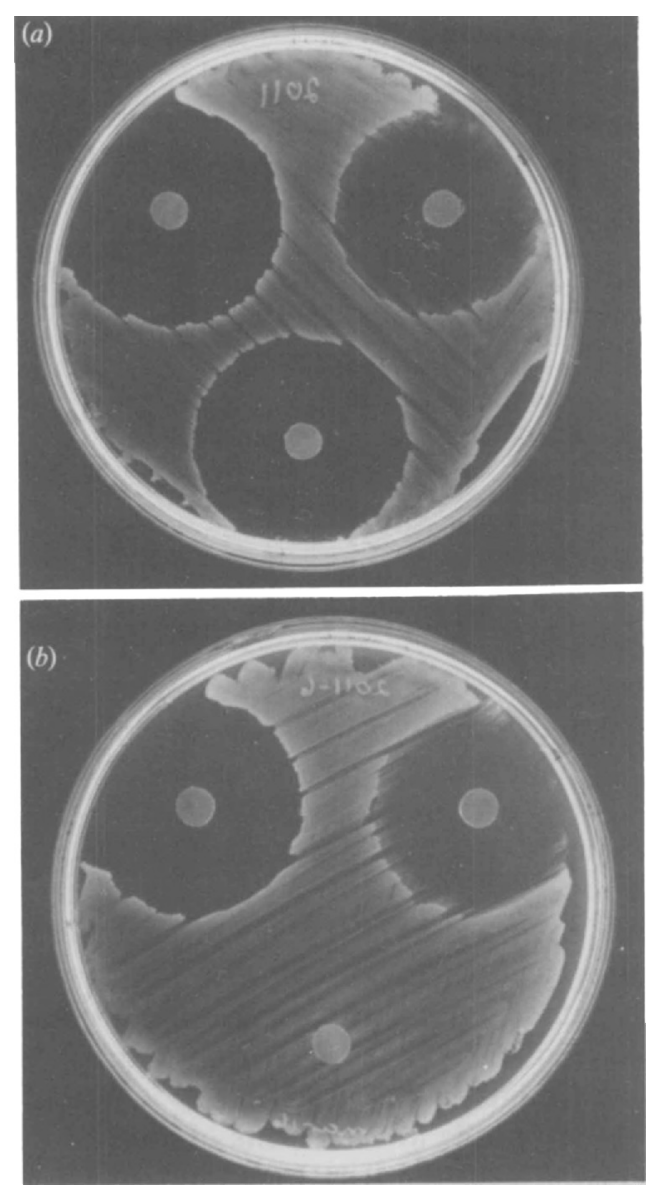

Fig. 1. Conversion of phenyllactate to phenylpyruvate in vivo. The agar plates contained defined, glucose-based medium and were spread with confluent lawns of ATCC 27628 (a) or ATCC 27628-1 (b). Sterile $6 \mathrm{~mm}$ paper discs placed on the agar surface contained phenylpyruvate (left), L-phenylalanine (right), or phenyllactate (bottom), each at $0.4 \mu \mathrm{mol}$ per disc. Photographs were taken after $2 \mathrm{~d}$ incubation at $37^{\circ} \mathrm{C}$.

reverse the inhibition of growth. Since iLDH converts 4-hydroxyphenyllactate to 4-hydroxyphenylpyruvate, growth inhibition of ATCC 27628 by L-phenylalanine is also reversed by 4-hydroxyphenyllactate. Fig. 3(a) illustrates the ability of L-tyrosine, 4-hydroxyphenylpyruvate, and 4-hydroxyphenyllactate to reverse growth inhibition caused by L-phenylalanine in ATCC 27628. In contrast, mutant ATCC 27628-1 was unable to utilize 4-hydroxyphenyllactate for reversal of phenylalaninemediated inhibition of growth, but, as expected, 4hydroxyphenylpyruvate and L-tyrosine were as effective in reversing growth inhibition as in the parent strain (Fig. $3 b$ ). Unlike the parent, the mutant could not utilize 4-hydroxyphenyllactate to reverse growth-inhibitory effects of tyrosine analogues such as $m$-fluorotyrosine (data not shown).

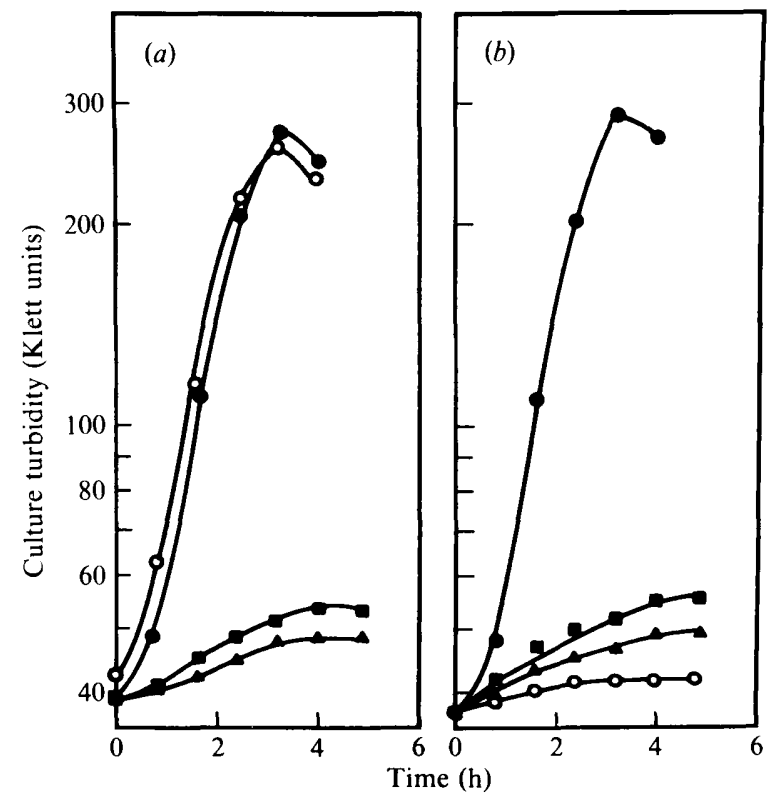

Fig. 2. Resistance of mutant ATCC 27628-1 to growth inhibition by phenyllactate. Growth-curve determinations for the mutant, ATCC 27628-1 (a), and the parent, ATCC 27628 (b), were carried out at $37^{\circ} \mathrm{C}$ as described in Methods. No additions: $\mathbf{\square},+100 \mu \mathrm{g} \mathrm{L}-$ phenylalanine $\mathrm{ml}^{-1} ; \triangle,+100 \mu \mathrm{g}$ phenylpyruvate $\mathrm{ml}^{-1} ; \mathrm{O},+100 \mu \mathrm{g}$ phenyllactate $\mathrm{ml}^{-1}$.

\section{Loss of iLDH in phenyllactate-resistant mutants}

Three phenyllactate-resistant mutants were assayed for iLDH activity using solubilized membranes, and none of them exhibited detectable activity. Data shown in Table 1 for mutant ATCC 27628-1 are representative of the three mutants. In all cases, pyruvate reductase activity $(\mathrm{nLDH})$ in the cytoplasmic fraction remained intact.

\section{Loss of coupled lactate oxidation and electron transport}

The ability of wild-type $N$. gonorrhoeae to reduce electron-transport components in the presence of lactate, phenyllactate or 4-hydroxyphenyllactate is shown in Fig. 4. Room-temperature difference spectra of dithionitereduced minus ferricyanide-oxidized components produced absorbance maxima in the alpha and beta region (i.e. $554 \mathrm{~nm}$ and $523 \mathrm{~nm}$, respectively), as shown in Fig. 4 (spectrum 4). Difference spectra were first recorded by use of a sample oxidized by sparging with air and then adding ferricyanide. The higher absorbance values of the air-oxidized samples in relation to ferricyanide-oxidized samples (spectrum 1, Fig. 4) is the result of a more complete oxidation of the respiratory-chain components, especially those which are not oxidized in the presence of oxygen. DL-Lactate or succinate increased the steady- 
Table 1. Activities of iLDH and nLDH in phenyllactate-resistant mutant ATCC 27628-1 and its parent ATCC 27628

\begin{tabular}{lccccc}
\hline & \multicolumn{4}{c}{ Enzyme activity* } \\
\cline { 2 - 5 } & Substrate $\dagger \ldots$ Lactate & Phenyllactate & $\begin{array}{c}\text { 4-Hydroxy- } \\
\text { phenyllactate }\end{array}$ & Pyruvate \\
\cline { 2 - 5 } Strain & 242 & 55 & 55 & 21 \\
ATCC 27628 & 0 & 0 & 0 & 23 \\
ATCC 27628-1 & & (iLDH) \\
\hline \hline
\end{tabular}

* The specific activity of iLDH is expressed as nmol 3-(4,5-dimethylthiazol-2-yl)-2,5-diphenyltetrazolium bromide reduced $\min ^{-1}\left(\mathrm{mg}^{2} \text { protein }\right)^{-1}$; the specific activity of $\mathrm{nLDH}$ is expressed as nmol NADH oxidized $\min ^{-1}$ (mg protein $)^{-1}$.

$\dagger$ DL mixtures of lactate, phenyllactate and 4-hydroxyphenyllactate were compared.
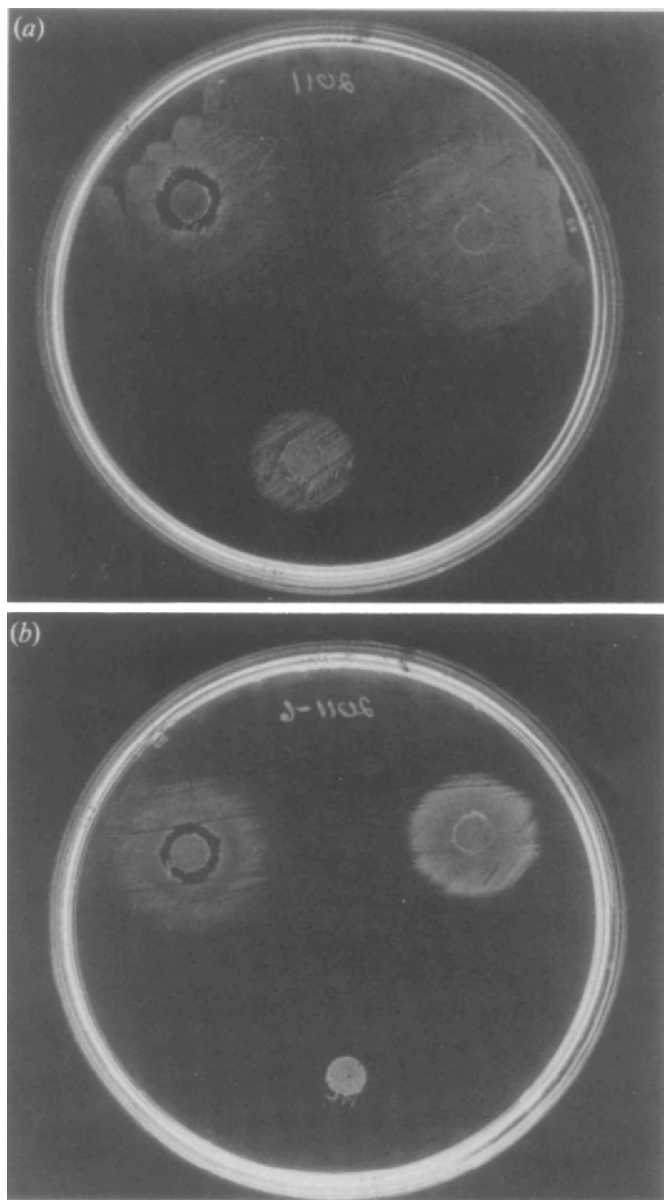

state levels of reduced cytochromes. The rate of reduction of cytochromes in the particulate material by lactate or phenyllactate (inset) was followed as an increase in $A_{554}$ relative to $A_{600}$. The rate of reduction of cytochromes was lower in the presence of phenyllactate, and the steady-state level of reduced cytochromes was about $50 \%$ of the values obtained with lactate as electron donor. Identical results were obtained with 4-hydroxyphenyllactate as with phenyllactate (data not shown). The $\Delta A_{523}$ in the presence of these various electron donors was comparable to the $\Delta A_{554}$.

In contrast to the above results obtained with wildtype ATCC 27628, the iLDH-deficient ATCC 27628-1 lacked the ability to reduce electron-transport components in the presence of lactate, phenyllactate or 4hydroxyphenyllactate. As expected, the reduction obtained with succinate and with dithionite resembled that obtained with the parent strain.

\section{Effect of iLDH deficiency upon carbon-source utilization}

Mutant ATCC 27628-1 retained normal ability to grow on lactate as the sole source of carbon. A comparison of growth rates obtained on glucose, pyruvate and lactate gave results similar to those obtained with the parent strain ATCC 27628. Growth results obtained on solid medium revealed that the mutant had acquired the ability to grow (poorly) on acetate, a characteristic not seen in the parent strain ATCC 27628.

Fig. 3. Conversion of 4-hydroxyphenyllactate to 4-hydroxyphenylpyruvate in vivo. Agar plates containing defined, glucose-based medium and $1 \mathrm{mM}$-L-phenylalanine were spread with confluent lawns of ATCC $27628(a)$ or ATCC 27628-1 (b). Sterile $6 \mathrm{~mm}$ paper discs placed on the agar surface contained L-tyrosine (right), 4-hydroxyphenylpyruvate (left), or 4-hydroxyphenyllactate (bottom), each at $0.4 \mu \mathrm{mol}$ per disc. Photographs were taken after $2 \mathrm{~d}$ incubation at $37^{\circ} \mathrm{C}$.

\section{Discussion}

The stimulation of oxygen consumption by gonococcal populations that use host-derived lactate to drive the electron-transport process has been demonstrated (Britigan et al., 1988). Since 4-hydroxyphenyllactate is present 


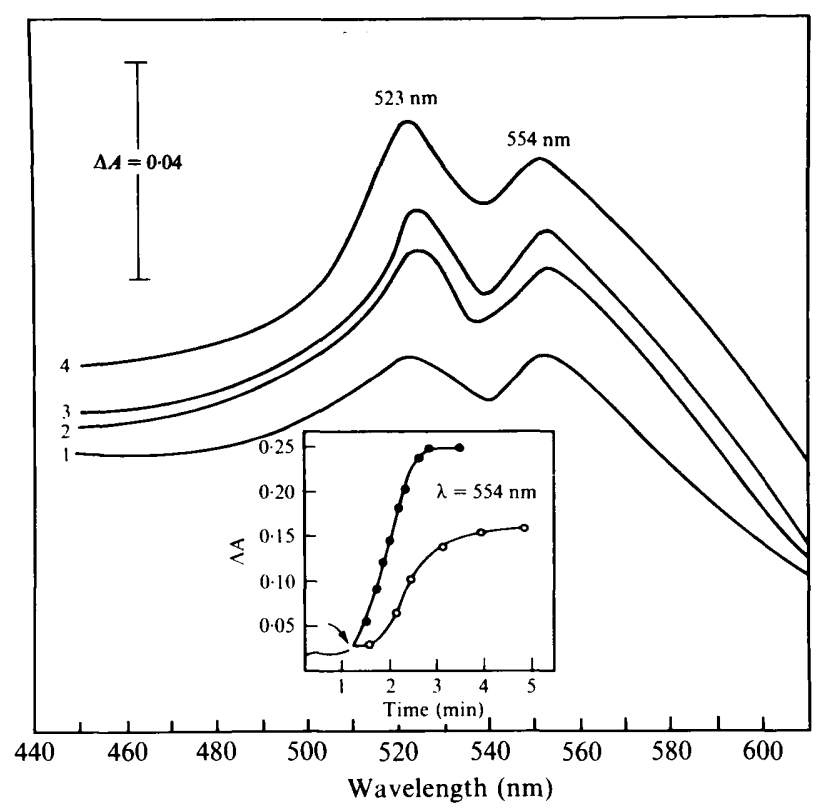

Fig. 4. Reduction of cytochromes in cell-membrane fragments from $N$. gonorrhoeae ATCC 27628 in the presence of lactate or phenyllactate. Difference spectrum 1 was obtained by first sparging the sample with air and then adding ferricyanide. Difference spectra 2, 3 and 4 were obtained in samples $\left(0.9 \mathrm{mg}\right.$ protein $\left.\mathrm{ml}^{-1}\right)$ where cytochromes were maximally oxidized by sparging with air followed by oxidation with ferricyanide, then reduced with lactate (2), reduced with succinate, (3) or reduced with dithionite (4). The inset shows the time course of reduction following addition (at the time indicated by the arrow) of lactate $(O)$ or phenyllactate $(O)$. The sample contained $8.1 \mathrm{mg}$ protein $\mathrm{ml}^{-1}$. See text for details.

in human tissues, we have suggested (Bhatnagar et al., $1989 a$ ) that this may provide a selective basis for the broadened substrate specificity of gonococcal iLDH. Membrane-bound cytochromes have been shown to be reduced in the presence of lactate (Winter \& Morse, 1975), and iLDH has been cited as a component of the respiratory chain (Morse, 1979). A prime function of iLDH may be to couple the assimilation of lactate to the ultimate generation of anaerobic conditions in the cell.

iLDH is unidirectional, catalysing only lactate oxidation. nLDH is also thought to be unidirectional in vivo, operating exclusively as a pyruvate reductase (Garvie, 1980). Since iLDH-deficient mutants assimilate lactate as sole carbon source, it is likely that under abnormal conditions $\mathrm{nLDH}$ is capable of functioning in the reverse-of-normal direction. In vitro properties of catalysis (Bhatnagar et al., 1989a) are consistent with expectations that pyruvate metabolism could pull the nLDH-mediated reaction in the direction of lactate oxidation readily. However, the inability of mutants to oxidize phenyllactate indicates that the nLDH lacks the broad specificity of the iLDH and could not use phenyllactate as a substrate. Apparently, no precedent is documented in the literature for in vivo operation of $\mathrm{nLDH}$ in the direction of lactate oxidation.

Since iLDH-deficient mutants lose the ability to use both D-lactate and L-lactate, we conclude that a single iLDH species exists which is not stereospecific. This is consistent with enzymological data recently reported (Bhatnagar et al., 1989a).

A phenotypic property of mutant ATCC 27628-1, not seen in the parent strain, was the gain in ability to assimilate acetate as sole source of carbon. The inability of the parent strain ATCC 27628 to utilize acetate could be the consequence of lack of malate oxidase. The quinone pool may be rapidly reduced by NADH dehydrogenase, using the reducing power generated from glucose and pyruvate metabolism. Malate oxidase, a flavoprotein, must be coupled with the electrontransport chain for activity (Holten \& Jyssum, 1974). If the quinone pool is rapidly reduced via the activity of iLDH, the absence of malate oxidase may prevent the oxidation of acetate. If absence of iLDH in the mutant does not permit as complete a reduction of the quinone pool, then malate oxidase may be in the oxidized state. An active malate oxidase (as well as aconitase and isocitrate dehydrogenase) could then allow oxidation of acetate in the iLDH-deficient mutant. Elucidation of the nature of iLDH with respect to the complexity of carbon flow and electron transport in this unique organism should be of great interest.

This work was supported by grant DK-38309 (to R. A. J.) from the United States Public Health Service and by a grant (to A.T.H.) from the Hamilton Civic Hospital Research Fund (Hamilton, Ontario).

This paper is Florida Agricultural Station Journal series no. R-00272.

\section{References}

Bhatnagar, R. K., Hendry, A. T., Shanmugam, K. T. \& Jensen, R. A. $(1989 a)$. The broad-specificity, membrane-bound lactate dehydrogenase of Neisseria gonorrhoeae : ties to aromatic metabolism. Journal of General Microbiology 135, 353-360.

Bhatnagar, R. K., Berry, A., Hendry, A. T. \& Jensen, R. A. $(1989 \mathrm{~b})$. The biochemical basis for growth inhibition by L-phenylalanine in Neisseria gonorrhoeae. Molecular Microbiology 3, 429-436.

BRADFORD, M. M. (1976). A rapid and sensitive method for the quantitation of microgram quantities of protein utilizing the principle of protein-dye binding. Analytical Biochemistry 72, 248-254.

Britigan, B. E., Klapper, D., Svendsen, T. \& Cohen, M. S. (1988). Phagocyte derived lactate stimulates oxygen consumption by Neisseria gonorrhoeae: an unrecognized aspect of the oxygen metabolism of phagocytosis. Journal of Clinical Investigation 81, 318-324.

GARVIE, E. (1980). Bacterial lactate dehydrogenases. Microbiological Reviews 44, 106-139.

HENDRY, A. T. (1983). Growth responses of Neisseria gonorrhoeae auxotypes to required amino acids and bases in liquid medium. Canadian Journal of Microbiology 29, 1309-1313.

HENDRY, A. T. \& DilloN, J. R. (1984). Growth inhibition of Neisseria gonorrhoeae isolates by L-phenylalanine and its analogues in defined media. Canadian Journal of Microbiology 30, 1319-1325. 
Holten, E. \& Jyssum, K. (1974). Activities of some enzymes concerning pyruvate metabolism in Neisseria. Acta Pathologica et Microbiologica Scandinavica B82, 843-848.

MoRse, S. A. (1979). The biology of gonococcus. Critical Reviews in Microbiology 7, 93-109.

Weiner, J. H. \& Heppel, L. A. (1972). Purification of the membrane- bound pyridine nucleotide independent L-glycerol 3-phosphate dehydrogenase from Escherichia coli. Biochemical and Biophysical Research Communications 47, 1360-1385.

WINTER, D. B. \& MoRse, S. A. (1975). Physiology and metabolism of pathogenic Neisseria: partial characterization of the respiratory chain of Neisseria gonorrhoeae. Journal of Bacteriology 123, 631-636. 